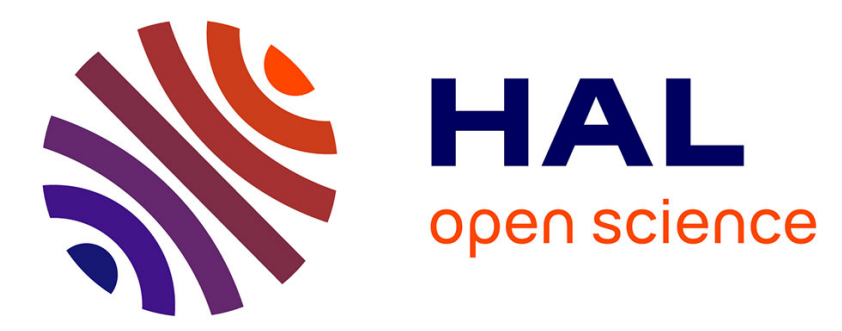

\title{
Photon Statistics of Incoherent Cathodoluminescence with Continuous and Pulsed Electron Beams
}

Magdalena Solà-Garcia, Kelly W Mauser, Matthias Liebtrau, Toon Coenen, Silke Christiansen, Sophie Meuret, Albert Polman

\section{- To cite this version:}

Magdalena Solà-Garcia, Kelly W Mauser, Matthias Liebtrau, Toon Coenen, Silke Christiansen, et al.. Photon Statistics of Incoherent Cathodoluminescence with Continuous and Pulsed Electron Beams. ACS photonics, 2021, 8 (3), pp.916 - 925. 10.1021/acsphotonics.0c01939 . hal-03437299

\section{HAL Id: hal-03437299 \\ https://hal.science/hal-03437299}

Submitted on 19 Nov 2021

HAL is a multi-disciplinary open access archive for the deposit and dissemination of scientific research documents, whether they are published or not. The documents may come from teaching and research institutions in France or abroad, or from public or private research centers.
L'archive ouverte pluridisciplinaire HAL, est destinée au dépôt et à la diffusion de documents scientifiques de niveau recherche, publiés ou non, émanant des établissements d'enseignement et de recherche français ou étrangers, des laboratoires publics ou privés. 


\title{
Photon Statistics of Incoherent Cathodoluminescence with Continuous and Pulsed Electron Beams
}

\author{
Magdalena Solà-Garcia,* Kelly W. Mauser, Matthias Liebtrau, Toon Coenen, Silke Christiansen, \\ Sophie Meuret, and Albert Polman
}

Cite This: ACS Photonics 2021, 8, 916-925

Read Online

ACCESS | Llll Metrics \& More | 国 Article Recommendations | sl Supporting Information

ABSTRACT: Photon bunching in incoherent cathodoluminescence (CL) spectroscopy originates from the fact that a single high-energy electron can generate multiple photons when interacting with a material, thus, revealing key properties of electron-matter excitation. Contrary to previous works based on Monte Carlo modeling, here we present a fully analytical model describing the amplitude and shape of the second order autocorrelation function $\left(g^{(2)}(\tau)\right)$ for continuous and pulsed electron beams. Moreover, we extend the analysis of photon bunching to ultrashort electron pulses, in which up to 500 electrons per pulse excite the sample within a few picoseconds. We obtain a simple equation relating the bunching strength $\left(g^{(2)}(0)\right)$ to the

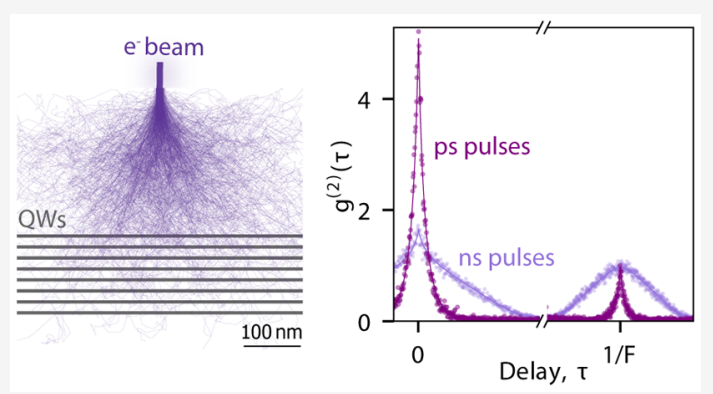
electron beam current, emitter decay lifetime, pulse duration, in the case of pulsed electron beams, and electron excitation efficiency $(\gamma)$, defined as the probability that an electron creates at least one interaction with the emitter. The analytical model shows good agreement with the experimental data obtained on InGaN/GaN quantum wells using continuous, ns-pulsed (using beam blanker) and ultrashort ps-pulsed (using photoemission) electron beams. We extract excitation efficiencies of 0.13 and 0.05 for 10 and $8 \mathrm{keV}$ electron beams, respectively, and we observe that nonlinear effects play no compelling role, even after excitation with ultrashort and dense electron cascades in the quantum wells.

KEYWORDS: cathodoluminescence, photon statistics, autocorrelation measurements, bunching, quantum wells

$\mathrm{P}$ hoton statistics in incoherent cathodoluminescence (CL) reveals fundamental properties of the interaction of highenergy electrons $(\sim 1-300 \mathrm{keV})$ with matter. ${ }^{1}$ In particular, the second-order autocorrelation function $\left(g^{(2)}(\tau)\right)$ exhibits strong bunching $\left(g^{(2)}(0) \gg 1\right)$ when exciting a material, such as a semiconductor or insulator, with an electron beam, ${ }^{1-3}$ contrary to conventional photoluminescence measurements with laser excitation (typically $g^{(2)}(0)=1$ ). ${ }^{4}$ This is because each electron initially excites bulk plasmons in the material, which end up generating thermalized carriers that diffuse and recombine. This recombination can lead to either the emission of a photon with energy corresponding to the bandgap of the material (bimolecular recombination) or to the excitation of another emitter embedded in the material, such as a defect or quantum well, which can then decay radiatively. Either cases can result in the emission of multiple photons per incoming electron. ${ }^{5}$ Recently, $g^{(2)}(\tau)$ measurements have been used to quantify the excitation efficiency of electrons in $\mathrm{InGaN} / \mathrm{GaN}$ quantum wells (QWs) with different geometries. ${ }^{6,7}$ The excitation efficiency is defined as the probability of an electron to interact with the emitter, in that case, the QWs. Moreover, the $g^{(2)}(\tau)$ technique allows to extract the emitter decay dynamics without the need of a pulsed electron beam. ${ }^{1}$ These new insights into the use of $g^{(2)}(\tau)$ measurements in CL are key for a complete and quantitative analysis of electron microscopy experiments.

All CL bunching experiments performed so far have been described using Monte Carlo-based numerical models, showing good agreement with the measured $g^{(2)}(\tau)$ curves and the dependence of $g^{(2)}(0)$ on the electron current. However, Monte Carlo models are time-consuming and fail to provide a full understanding of the bunching process and the key parameters that determine its amplitude. Moreover, fitting the experimental data with a Monte Carlo model is complex and requires additional computation and interpolation procedures, thus making the $g^{(2)}(\tau)$ analysis less accessible.

In addition to this, so far, CL autocorrelation measurements have been limited to the cases of continuous and ns-pulsed electron beams. Recently, ultrafast electron microscopy using fs-ps electron pulses as excitation sources has emerged as a powerful technique to access the dynamics of electron excitation of materials with high temporal resolution,

Received: December 22, 2020

Published: February 11, 2021 

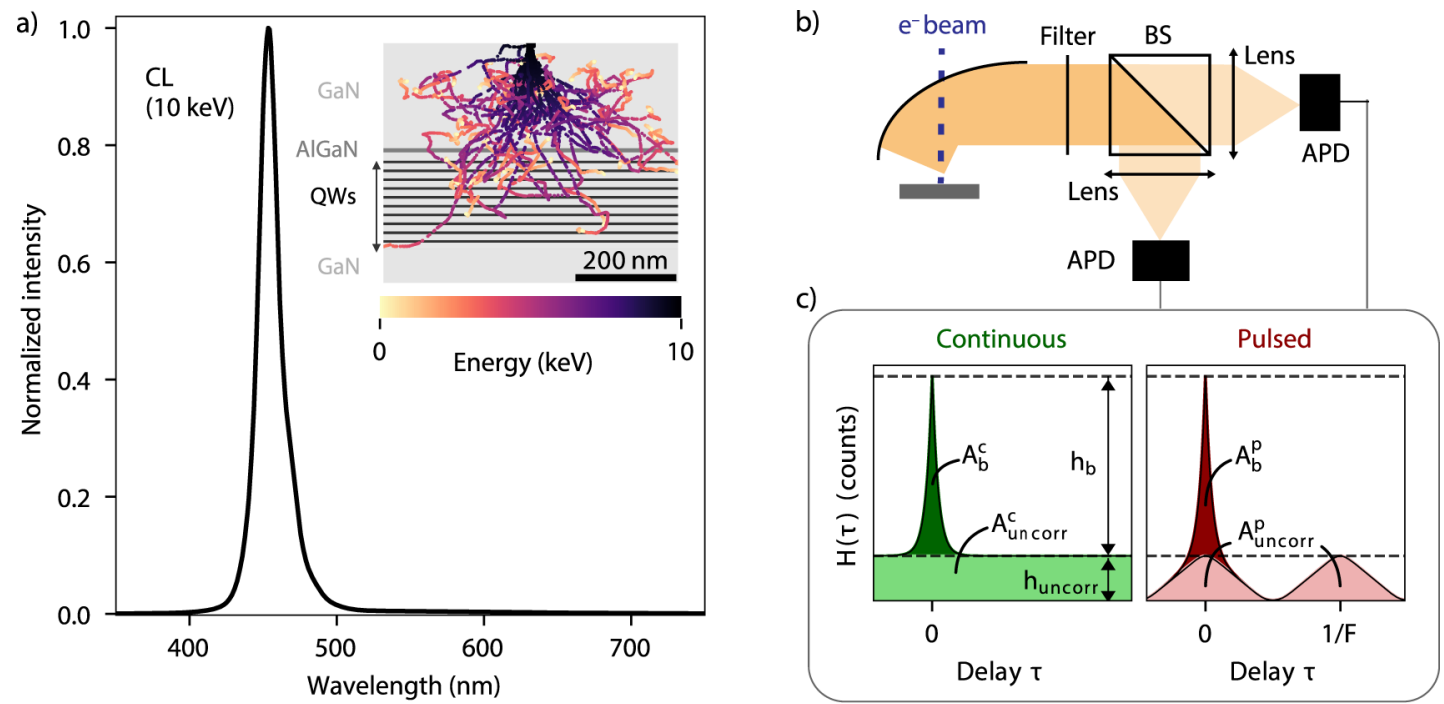

Figure 1. (a) Cathodoluminescence (CL) spectrum of InGaN/GaN quantum wells (QWs) obtained with a continuous $10 \mathrm{keV}$ beam (242 pA). Inset: schematic of the InGaN/GaN heterostructure overlaid with the results from Monte Carlo simulations of the electron trajectory inside the sample, perfomed using the Casino software. ${ }^{21}$ Each curve indicates the trajectory of one electron, and the color bar indicates the energy of the electron at each position. (b) Schematic of the CL collection and analysis using a Hanbury-Brown and Twiss (HBT) experiment. (c) Schematic of the expected histograms obtained using the HBT experiments using continuous (left) and pulsed (right) electron beams.

combined with the nanoscale electron-beam spatial resolution. ${ }^{8-10}$ Ultrafast electron microscopy has already been used to study electron-generated carrier dynamics ${ }^{11,12}$ and phase transitions, ${ }^{13,14}$ among others. Additionally, the development of techniques such as photon-induced near-field microscopy (PINEM) has exploited the quantum nature of the electron wave packet, ${ }^{15,16}$ thus, leading the way to the study of quantum-mechanical aspects of electron-light-matter interactions inside an electron microscope. Autocorrelation measurements, such as $g^{(2)}(\tau)$, using ultrashort electron pulses can offer new insights into the dynamics of the excitation of a material with dense electron pulses. ${ }^{17}$

In this paper, we resolve the above-mentioned limitations of current $g^{(2)}(\tau)$ analyses that use Monte Carlo simulations. We develop a fully analytical model to describe the value of $g^{(2)}(0)$ as a function of four experimental parameters for three different electron beam configurations. Our analytical model describes how the electron current (or number of electrons per pulse), emitter lifetime, excitation efficiency, and pulse duration, in the case of pulsed electron beams, determine the value of $g^{(2)}(0)$. Using our analysis, we directly extract the electron excitation efficiency $\gamma$, defined as the fraction of electrons that create at least one interaction with the emitter, ${ }^{6}$ from one simple equation. We also show that our model reproduces the Monte Carlo simulations developed in previous work.

In order to further test the validity of the model, we perform $g^{(2)}(\tau)$ experiments on InGaN/GaN quantum wells with both continuous and pulsed electron beams. In particular, we study two types of pulsed electron beams: with relatively long (up to $200 \mathrm{~ns}$ ) and ultrashort (a few ps) pulse durations. In the case of ultrashort pulses, we vary the number of electrons per pulse from (on average) less than 1 up to $~ 500$, thus, allowing us to access regimes in which several electrons interact with the sample within the bulk plasmon decay and carrier thermalization time scales. Here, our analytical model shows that $g^{(2)}(0)$ depends only on the number of electrons per pulse and the excitation efficiency. From the model it can also be derived that, in the case of a pulsed electron beam, the excitation efficiency can be obtained alternatively through a simple analysis, without the need of any fitting procedure. Our analysis of the $g^{(2)}(\tau)$ function shows that even for dense cascades generated by 500 electrons per pulse (i.e., within a few picoseconds) nonlinear effects do not have a compelling contribution in the excitation and carrier recombination of InGaN/GaN QWs.

\section{EXPERIMENTAL SECTION}

Cathodoluminescence experiments are performed in a scanning electron microscope (SEM) equipped with a parabolic mirror that collects the emitted light. The statistics of CL emission are analyzed using a Hanbury-Brown and Twiss (HBT) geometry, ${ }^{18}$ composed of a 50:50 beam splitter (BS) and two avalanche photodiodes (APDs) as single-photon counting detectors (Figure 1b). Experiments with varying pulse widths (6-200 ns) are performed using an electrostatic electron beam blanker. Ultrashort pulses, with pulse widths of a few picoseconds, are obtained by focusing a fs-laser $(\lambda=258$ $\mathrm{nm}$ ) onto the electron cathode, inducing photoemission of electron pulses. ${ }^{19,20}$ All of our experiments are performed at room temperature.

We study a bulk semiconductor heterostructure of InGaN/ GaN quantum wells, grown by molecular beam epitaxy. ${ }^{6} \mathrm{~A}$ schematic of the structure is shown in the inset of Figure 1a. The sample consists of $102 \mathrm{~nm}$ thick InGaN layers, separated with $15 \mathrm{~nm} \mathrm{GaN}$ layers. A $2 \mathrm{~nm}$ AlGaN barrier layer is grown on top of the quantum well stack, and the whole structure is buried below a $250 \mathrm{~nm}$ thick p-type $\mathrm{GaN}$ layer. The substrate is composed of n-type GaN. The inset also shows the results of Monte Carlo simulations of the trajectory of a $10 \mathrm{keV}$ electron beam inside the sample, performed using the Casino software. ${ }^{21}$ Each dot in the plot represents a collision of the primary electron with the sample, which can lead to the excitation of one or more bulk plasmons. The color of the dot indicates the energy of the primary electron beam at that point. The results show that only a small fraction of the electrons will 
directly reach the QWs, as previously shown using $g^{(2)}(\tau)$ measurements. ${ }^{6}$ Moreover, the AlGaN layer acts as a carrier blocking layer, ${ }^{22}$ hence, only carriers generated below this layer can excite the QWs.

Figure 1a shows a typical CL spectrum obtained when exciting the sample with $10 \mathrm{keV}$ electrons. The emission originates mostly from the QWs, corresponding to the InGaN band edge emission peak around $450 \mathrm{~nm}$. Defect luminescence from the yellow band, ${ }^{23,24}$ in the 520-650 spectral range, is barely visible in the spectrum, given that the intensity in this range is 30 times lower than the $\mathrm{QW}$ emission. This is in accordance with previous CL measurements on this sample, ${ }^{6}$ and is attributed to the fact that $10 \mathrm{keV}$ electrons do not reach the GaN substrate, thus, limiting the excitation of carriers in the bulk GaN. In the HBT experiments we use a bandpass filter $(450 \pm 40 \mathrm{~nm})$ to ensure that only the CL emission from the QWs is recorded.

\section{CONTINUOUS ELECTRON BEAM}

A typical $g^{(2)}(\tau)$ experiment consists of the acquisition of a histogram $(H(\tau))$ of the number of coincidence events (i.e., a correlation) as a function of the delay between two recorded photons $(\tau)$. A schematic of the recorded histogram obtained after excitation with a continuous electron beam is shown in Figure 1c (left). In this case, the $g^{(2)}(\tau)$ curve is obtained by normalizing the histogram with respect to the value at very long delay $(\tau \rightarrow \infty), h_{\text {uncorr }}$, which represents the amplitude for uncorrelated events. Hence,

$$
g^{(2)}(0)=\frac{H(0)}{H(\tau \rightarrow \infty)}=1+\frac{h_{\mathrm{b}}^{c}}{h_{\text {uncorr }}^{c}}
$$

where $h_{\mathrm{b}}^{c}$ is the amplitude of the bunching peak, as depicted in Figure 1c. $g^{(2)}(0)$ can be interpreted as the likelihood of having two photons with delay $\tau=0$ compared to any other delay. In the case of Poissonian statistics, such as for coherent light, $g^{(2)}(\tau)=1$ for any delay, ${ }^{4}$ while $g^{(2)}(0)<1$ indicates subPoissonian statistics (antibunching), as in the case of a singlephoton emitter, ${ }^{25,26}$ while $g^{(2)}(0)>1$ represents superPoissonian statistics (bunching). Some examples of processes in which bunching occurs are blackbody radiation ${ }^{27,28}$ and superradiance, ${ }^{29,30}$ as well as the CL emission presented here. From a statistical point of view, $h_{\mathrm{b}}$ is related to the total number of correlations (defined as the detection of two photons) leading to bunching, that is, coming from the same electron, while $h_{\text {uncorr }}^{c}$ represents the uncorrelated events, that is, correlations between photons that are generated by different electrons.

The temporal decay of the bunching peak is determined by the radiative decay of the emitter and enables determination of the emitter lifetime, as will be explained below. The area of the bunching peak, related to the height as $A_{\mathrm{b}}^{c}=\alpha_{\mathrm{b}} h_{\mathrm{b}}^{c}$, is proportional to the average number of possible combinations between pairs of photons that lead to bunching, that is, that come from the same electron. Here we have defined $\alpha_{\mathrm{b}}$ as the shape factor of the bunching peak. Similarly, the area $A_{\text {uncorr }}^{c}$ is related to the mean number of possible combinations of photon correlations from different electrons, that is, uncorrelated events, during the acquisition time of the experiment $T=$ $B t_{B}$. Here $B$ is the total number of bins in the experiment and $t_{B}$ is the time of each bin. From this it follows that $A_{\text {uncorr }}^{c}=$ $h_{\text {uncorr }}^{c}(2 B+1) t_{B} / 2$. The factor $2 B+1$ comes from the fact that the $g^{(2)}(\tau)$ histogram is theoretically built over positive and negative times, in a symmetric fashion. The additional factor 2 in the denominator accounts for the fact that the number of possible events decreases for increasing delay following a triangular function (see S2f in the Supporting Information). Taking these definitions into account, eq 1 becomes

$$
g_{\text {cont }}^{(2)}(0)=1+\frac{A_{\mathrm{b}}^{c}}{A_{\text {uncorr }}^{c}} \frac{(2 B+1) t_{B}}{2 \alpha_{\mathrm{b}}}
$$

The model is constructed following the subsequent steps that start with an electron entering the material, until a photon is emitted, similar to the previous Monte Carlo model. ${ }^{1,6}$ The steps are as follows:

1. Excitation of $b_{i}$ bulk plasmons in GaN close to the QWs, described by a Poisson distribution with expected value $b$. It should be noted that the number of plasmons generated per electron will probably be larger than $b_{i}$, but here we only consider those that can create carriers which can excite the QWs, that is, excited within the carrier diffusion length.

2. Decay of each bulk plasmon into $m_{i}$ thermalized carriers, again described by a Poisson distribution with expected value $m$.

3. Diffusion of carriers, which can either (a) excite a QW, which emits a photon. The joint probability of these two steps is accounted in the parameter $\eta$, or (b) not excite a QW or excite a QW which then decays nonradiatively.

This step is assumed to follow a binomial distribution, with $m_{i}$ representing the number of events and $\eta$ probability that an event results in the emission of a photon.

We should note that here we refer to QW excitation, but the model can be applied to any other kind of emitter, or simply to carrier recombination. In the case of a thin sample and high electron energy, such as in transmission electron microscopy (TEM) experiments, the average number of bulk plasmons (b) defined in step 1 corresponds to the ratio between the thickness and the electron mean free path. ${ }^{1,31}$ In the case of thicker samples, $b$ takes into account the probability that carriers and, in particular, minority carriers, created after electron excitation actually reach the emitter. It therefore depends on the sample geometry and diffusion length of minority carriers, as will be seen further in the text.

A key aspect of our $g^{(2)}(0)$ model is that it accounts for the combined stochastic nature of all the involved processes. The model is therefore based on the calculation of the average possible combinations of pair-correlation events that lead to bunching $\left(A_{\mathrm{b}}^{c}\right)$ and to uncorrelated events $\left(A_{\text {uncorr }}^{c}\right)$. A full derivation of the model is provided in the Supporting Information (S2). In brief, from step 2 and 3 we obtain that the average number of possible combinations of paircorrelation events created after the excitation of $b_{i}$ bulk plasmons is given by $b_{i}^{2} m^{2} \eta^{2}$. We then need to find the average number of combinations of correlations between pairs of photons from the same electron (i.e., ignoring pair-correlation events created by photons from different electrons), taking into account that $b_{i}$ follows a Poisson distribution. Given $n$ electrons arriving at the sample during the time of the experiment $T$, the average number of combinations of paircorrelation events leading to bunching becomes

$$
A_{\mathrm{b}}^{c}=n b(b+1) m^{2} \eta^{2}
$$

Similarly, it can be shown that the expected value of the number of combinations of pair-correlation events leading to 
uncorrelated events, that is, pairs of photons coming from different electrons, is (see S2b)

$$
A_{\text {uncorr }}^{c}=n(n-1) b^{2} m^{2} \eta^{2}
$$

We now insert eqs 3 and 4 into eq 2 , and rewrite $n$ as a function of the electron current, $I=n q /\left(B t_{b}\right)$, where $q$ is the electron charge. We also consider the limit $B \gg 1$, which is reasonable given that the acquisition time is typically minutes or more, while the time resolution is usually less than $1 \mathrm{~ns}$. We then obtain the following expression for the amplitude of $g^{(2)}(\tau)$ at 0 delay:

$$
g_{\text {cont }}^{(2)}(0)=1+\frac{q}{I \alpha_{\mathrm{b}}} \frac{b+1}{b}
$$

Several aspects are noticeable from eq 5. First of all, we can see that the value of $g^{(2)}(0)$ is inversely proportional to the electron beam current, which is in agreement with previous experimental results. ${ }^{1,2,6}$ This can now be understood from the fact that the amplitude of the bunching peak scales linearly with the number of electrons reaching the sample (eq 3), as it depends on the number of correlations between photons from the same electron. Instead, the background scales quadratically (eq 4), since it depends on the events created by photons from different electrons.

Second, eq 5 shows that $g^{(2)}(0)$ does not depend on the number of carriers generated per bulk plasmon $m$, nor on the efficiency of these carriers to excite a $\mathrm{QW}$ or the quantum efficiency of the QW (both processes included with $\eta$ ). The only relevant parameter is the number of bulk plasmons created close to the QWs $(b)$. This is in agreement with the fact that $g^{(2)}(\tau)$ measurements are independent of the absolute intensity incident on the detectors, as long as the statistics of the emission process is preserved. ${ }^{4}$ Notice that even in the case $b=1$, that is, on average one bulk plasmon per electron interacts with the QWs, $g^{(2)}(0)>1$ due to the stochastic nature of the plasmon excitation process. It follows from eq 5 that the bunching contribution to $g^{(2)}(0)$ increases with decreasing $b$, given that decreasing the number of interacting plasmons generated per electron would have a similar effect as decreasing the current. Given the Poissonian nature of the $b_{i}$ parameter, the average number of interacting bulk plasmons $b$ is related to the probability of creating at least one interaction (bulk plasmon) close to the emitter, ${ }^{6}$ defined as

$$
\gamma=1-\operatorname{Poiss}(0 ; b)=1-e^{-b}
$$

where $\gamma$ can be interpreted as the excitation efficiency of the electron in the given material geometry.

Finally, the value of $g^{(2)}(0)$ also depends on the shape of the bunching curve $\left(h_{\mathrm{b}}(\tau)\right)$, which is represented by the dependence on $\alpha_{\mathrm{b}}$. Given an emitter decay $y_{\text {emitter }}(t)$, it can be shown that the number of correlations between photons emitted with a delay $\tau$ is proportional to (see S4a in the Supporting Information)

$$
h_{\mathrm{b}}(\tau)=\int_{0}^{\infty} y_{\text {emitter }}(t) y_{\text {emitter }}(t+\tau) \mathrm{d} t
$$

In the case that the emitter decays as a simple exponential with lifetime $\tau_{\text {emitter }}, h_{\mathrm{b}}(\tau)$ is an exponential with $\tau_{\text {emitter, }}$ and thus, the decay of the $g^{(2)}(\tau)$ curve directly gives the emitter decay. In this case, the relation between the area and the height of the bunching peak is $\alpha_{\mathrm{b}}=2 \tau_{\text {emitter }}$ (see S2g in the Supporting Information). In the case of more complex decay mechanisms, we should apply eq 7 to extract the emission dynamics from the $g^{(2)}(\tau)$ measurement. Equations 5 and 7 can now be directly used to fit experimental data of $g^{(2)}(\tau)$ versus beam current, to determine $b$ and, hence, $\gamma$, thus, providing essential information on the electron beam excitation efficiency in incoherent $\mathrm{CL}$ excitation.

Figure 2a shows a selection of $g^{(2)}(\tau)$ measurements of the QW sample at different electron currents, all obtained using a
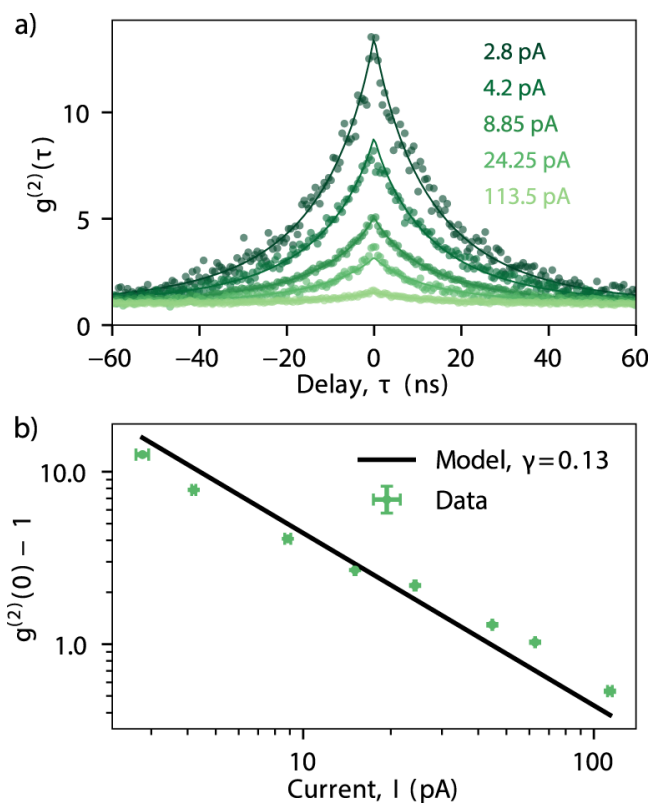

Figure 2. $g^{(2)}(\tau)$ measurements with a continuous electron beam. (a) $g^{(2)}(\tau)$ experiments obtained for different electron currents, with an electron energy of $10 \mathrm{keV}$. The points represent the data and the solid lines the fit. (b) Fits of $g^{(2)}(0)-1$ vs electron current obtained from the curves in (a). The black solid line is the fit obtained using eq 5 , from which a value of $\gamma=0.13$ is obtained. The error bars represent the uncertainty in the measured value of the electron current (horizontal) and fit errors (vertical).

continuous $10 \mathrm{keV}$ electron beam. The time binning in all measurements is set to $t_{b}=512 \mathrm{ps}$. At the lowest current $(2.8$ $\mathrm{pA}), g^{(2)}(0)=12.6$ is obtained, while the value of $g^{(2)}(0)$ strongly decreases for increasing current. The curves cannot be properly fitted with a simple exponential decay, probably due to multiple decay processes taking place simultaneously. Instead, the emitter decay $\left(y_{\text {emitter }}(t)\right)$ can be described with a stretched exponential, given by

$$
y_{\text {emitter }}(t)=y_{0} e^{-\left(t / \tau_{\text {emitter }}\right)^{\beta_{\text {emitter }}}}
$$

with parameters $\tau_{\text {emitter }}$ representing the average emitter lifetime and $\beta_{\text {emitter }}$ is the deviation from a pure exponential decay. ${ }^{32}$ This is further confirmed by direct measurements of the decay statistics of the QWs (see S4c). In this case, the shape of $g^{(2)}(\tau)$ does not give directly the emitter decay properties, but we need to fit the data with eq 7 , which can be solved numerically. The solid lines in the figures correspond to the fits, from which we obtain an emitter lifetime of $\tau_{\text {emitter }}=$ $10.65 \pm 0.32 \mathrm{~ns}$ and $\beta_{\text {emitter }}=0.76 \pm 0.01$.

In order to compare the experimental results with the analytical model, Figure $2 \mathrm{~b}$ shows the value of $g^{(2)}(0)-1$, obtained from the fits of each curve, as a function of electron current. The horizontal error bars indicate the uncertainty in 
measuring the electron current, while the vertical errors are derived from the fitting procedure. We observe that the value of $g^{(2)}(0)-1$ exhibits a linear decrease (on a log-log plot) with slope -1 , as predicted by eq 5 . The shape factor $\alpha_{\mathrm{b}}$ is calculated numerically from the solution of eq 7 using the derived values of $\tau_{\text {emitter }}$ and $\beta_{\text {emitter }}$, thus, becoming $\alpha_{\mathrm{b}}=25.04$. Therefore, we can extract $b$ from eq 5 . We obtain the best fit for $b=0.13$, which yields an excitation efficiency of $\gamma=0.13 \pm$ 0.01 , meaning that on average, only 13 out of 100 electrons actually interact with the QWs. This low interaction between the electrons and the QWs is attributed to the fact that at 10 $\mathrm{keV}$ most electrons lose their energy before arriving to the QWs, as shown in Figure $1 \mathrm{~b}$ and discussed in ref 6. Moreover, the carriers generated on the top GaN layer cannot reach the QWs, due to the presence of the AlGaN blocking layer on top of the QWs. For reference, we also show in the Supporting Information (section S1) that the results from the model are in excellent agreement with those obtained with the Monte Carlo-based approach proposed in previous works, ${ }^{1,6}$ confirming that our model serves as an effective analytical version of the Monte Carlo one.

\section{PULSED ELECTRON BEAM}

$g^{(2)}(\tau)$ experiments can also be performed using pulsed electron beams, which can offer advantages such as lower acquisition times and simpler analysis, as will be discussed below. In this configuration, the photon emission dynamics is shaped by the temporal spread of electrons, and thus, a modified model needs to be developed. A schematic of the histogram obtained in an HBT experiment in pulsed conditions is shown in Figure 1c (right). In contrast to the continuous case, here the histogram is composed of a peak at $\tau$ $=0$, containing correlations between photons from the same electron pulse, and peaks at delays corresponding to the time between pulses $\left(\tau_{i}=i / F\right.$, with $i$ being an integer number and $F$ the repetition rate). The latter correspond to correlations between photons from consecutive pulses $(i= \pm 1)$, from every second pulse $(i= \pm 2)$ and so on. These peaks are thus analogous to the background $\left(A_{\text {uncorr }}^{c}\right)$ in the continuous case.

The derivation of the model for the pulsed case is similar to the one for the continuous one, with the main difference being the shape factor of the bunching $\left(\tau_{0}\right)$ and uncorrelated $\left(\tau_{i}, i \neq\right.$ $0)$ peaks. Given that the peaks at $\tau_{i}(i \neq 0)$ contain correlations between photons from different pulses, their shape is determined by both the electron pulse and emitter decay, as explained in $\mathrm{S} 4$ in the Supporting Information. The ratio between the area $\left(A_{\mathrm{uncorr}, i}^{p}\right)$ and the height $\left(h_{\mathrm{uncor}, i}\right)$ of any of these peaks is given by $A_{\text {uncorr }, i}^{p}=\alpha_{\text {conv }} h_{\text {uncorr }, i} \cdot \alpha_{\text {conv }}$ is thus a shape factor, which will depend on the particular shape of the electron pulse and emitter decay.

The peak centered at $\tau=0$, accounts for correlations between photons from the same pulse, and has two components $\left(A_{0}^{p}=A_{\mathrm{uncorr}, 0}^{p}+A_{\mathrm{b}}^{p}\right)$. The first component corresponds to correlations between photons from the same pulse, but different electrons, and therefore has a shape factor $\alpha_{\text {conv }}$. The second component corresponds to correlations between photons from the same electron, which is what constitutes the bunching. Similar to the continuous case, we can consider that all the excitations take place instantaneously, given that the time scale of bulk plasmon decay and carrier diffusion (typically in the fs/ps regime) ${ }^{5}$ is much smaller than the emitter lifetime (hundreds of ps or ns). Therefore, the shape of the electron pulse does not play a role in this component, contrary to the case of the uncorrelated component. The time between photons is only determined by the emitter lifetime, and $A_{\mathrm{b}}^{p}=\alpha_{\mathrm{b}} h_{\mathrm{b}}^{p}$, where $h_{\mathrm{b}}^{p}$ is the height of this peak.

Taking into account the shape factors, and calculating the average number of possible combinations of pair-correlations events for bunching $\left(A_{\mathrm{b}}^{p}\right)$ and uncorrelated $\left(A_{\mathrm{uncorr}, i}^{p}\right)$ events in an analogous way as in the continuous case (see $S 3$ in the Supporting Information for a full derivation), we obtain that for pulsed excitation

$$
g_{\text {pulsed }}^{(2)}(0)=1+\frac{\alpha_{\text {conv }}}{\alpha_{\mathrm{b}}} \frac{b+1}{n_{e} b}
$$

We observe that the expression for $g^{(2)}(0)$ for a pulsed beam is very similar to the one for the continuous case (eq 5). Here, $g^{(2)}(0)$ is inversely proportional to the number of electrons per pulse, which is related to the electron beam current through $n_{e}$ $=I / q F$, with $F$ being the repetition rate. The dependence of $g^{(2)}(0)$ on the average number of bulk plasmons that interact with the sample $(b)$ is exactly the same as for a continuous electron beam, showing that the pulsed $g^{(2)}(\tau)$ measurement fundamentally probes the interaction of electrons with the sample in the same way. The main difference between the continuous and pulsed cases is the factor $\alpha_{\text {conv }}$ in the pulsed case, the $g^{(2)}(0)$ depends also on the shape of the electron pulse. From the derivation of $g^{(2)}(0)$ (section S3), it also follows that now we can simply divide the area of the peak at $\tau$ $=0$ by the area of any other peak at $\tau \neq 0$ to obtain the excitation efficiency:

$$
\frac{A_{\mathrm{b}}^{p}+A_{\mathrm{uncorr}, 0}^{p}}{A_{\mathrm{uncorr}, i}^{p}}=1+\frac{b+1}{n_{e} b}=1+\frac{1-\log (1-\gamma)}{n_{e} \log (1-\gamma)}
$$

In this case we do not need any fitting procedure nor prior knowledge of electron pulse shape or emitter decay, thus making the analysis even simpler. This becomes particularly useful when having small signal-to-noise ratios or nontrivial emitter decays or electron pulse shapes, in which cases fitting becomes challenging.

In order to test the model for the pulsed case, we performed experiments using an ultrafast beam blanker, in which a set of electrostatic plates is inserted inside the electron column. One of the plates is driven using a pulse generator, which is set to send a square signal with peak-to-peak voltage of $5 \mathrm{~V}$ and offset $2.5 \mathrm{~V}$, while the other plate is grounded. This configuration allows us to obtain effectively square electron pulses with pulse width $(\Delta p)$ determined by the repetition rate $F$ and duty cycle $D$. A characterization of the electron pulses is shown in the Supporting Information (S6a). In our experiments we kept the duty cycle fixed at $D=95 \%$ and varied the repetition rate from 0.2 to $6 \mathrm{MHz}$, resulting in pulse widths ranging from $200 \mathrm{~ns}$ down to 6 ns. Notice that an even smaller pulse width, down to $30 \mathrm{ps}$, can be obtained using the same ultrafast blanker in a different configuration, ${ }^{19}$ but long pulse widths were chosen to show their effect on the amplitude and shape of the bunching peak. The current in continuous mode (i.e., without blanking) was kept constant $(I=214 \mathrm{pA})$ for all experiments, therefore, changing the repetition rate results in a varying number of electrons per pulse, that is,

$$
n_{e}=\frac{I}{q} \frac{1-D / 100}{F}=\frac{I}{q} \Delta p
$$


Figure 3a shows a selection of $g^{(2)}(\tau)$ curves centered around the peak at delay 0 . The solid lines are the fits of the data,
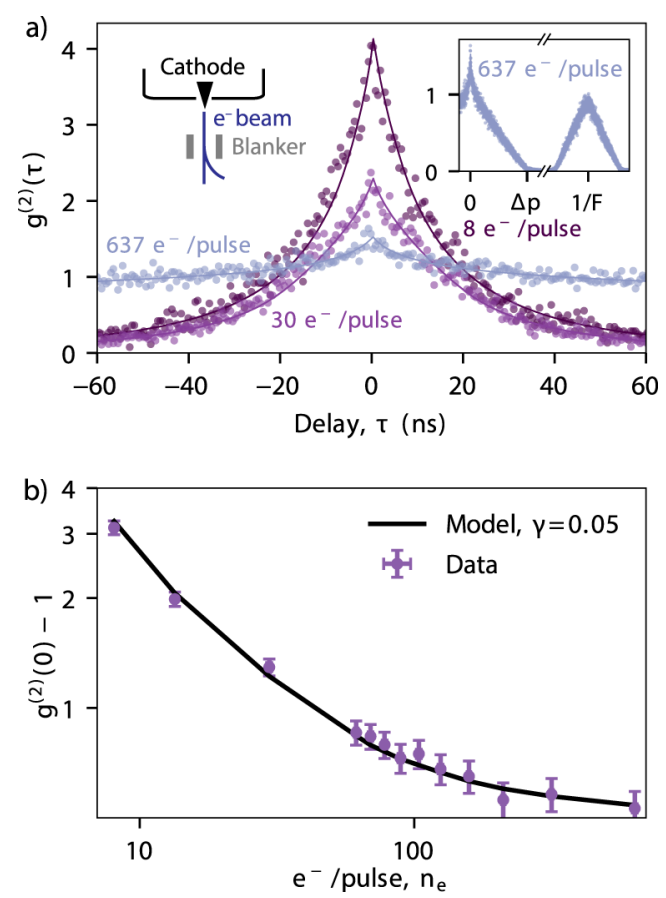

Figure 3. $g^{(2)}(\tau)$ measurements with a ns-pulsed electron beam. (a) $g^{(2)}(\tau)$ experiments obtained by changing the electron pulse width $\Delta p$, which leads to a change in the number of electrons per pulse. In this case the electron energy is $8 \mathrm{keV}$. The points represent the data and the solid lines the fit. Insets: (left) schematic of the beam blanking configuration, (right) example of a $g^{(2)}(\tau)$ measurement shown for a wider delay time range, thus showing the full peak at $\tau=0$ and the consecutive peak at $\tau=1 / F$, where $F$ is the repetition rate. (b) Experimental results of $g^{(2)}(0)-1$ vs number of electrons per pulse, obtained from the fits of the curves in (a). The black solid line corresponds to the best fit obtained using eq 9, which yields an excitation efficiency of $\gamma=0.05$. The error bars are derived from the uncertainty in the current measurement (horizontal) and fitting procedure (vertical).

which correspond to the sum of the solution from eq 7 (assuming a decay following a stretched exponential) and a convolution between a triangular curve and the same solution from eq 7. The triangular function comes from the convolution between two square pulses with pulse width $\Delta p$, representing two electron pulses (see S4 in the Supporting Information). The best fit of the curves is found for $\tau_{\text {emitter }}=5.40 \pm 0.33 \mathrm{~ns}$ and $\beta_{\text {emitter }}=0.56 \pm 0.01$. The difference between these values and the ones found in the continuous experiment (10.7 ns and 0.67 , respectively) is attributed to the inhomogeneity of the sample, which results in emission lifetimes that depend on sample position (see S8 in the Supporting Information). The discrepancy could also come from the fact that at $10 \mathrm{keV}$ we might be probing deeper QWs, which can exhibit different lifetimes. The curve at the lowest number of electrons per pulse $\left(8 \mathrm{e}^{-} /\right.$pulse $)$exhibits the highest amplitude $\left(g^{(2)}(0)=\right.$ $4.1)$. In this case, the pulse width (6 ns) is comparable to the emitter lifetime, and thus, no clear distinction between the bunching $\left(A_{b}^{p}\right)$ and uncorrelated $\left(A_{\mathrm{uncorr}, 0}^{p}\right)$ curves can be observed. Instead, the $g^{(2)}(\tau)$ curve for long pulses show a small sharp peak, corresponding to the bunching peak, on top of a broader background, as can be observed in the curve corresponding to $\Delta p=500 \mathrm{~ns}$ (637 electrons per pulse). The full shape of the uncorrelated peak can be observed in the right inset of Figure 3a, showing the peak around delay 0 and the first consecutive peak $\left(\tau_{1}=1 / F\right)$.

The value of $g^{(2)}(0)-1$ as a function of the number of electrons per pulse is shown in Figure $3 b$, which has been derived from the fits in Figure $3 \mathrm{a}$. We observe that the bunching decreases with increasing number of electrons per pulse, as expected, but contrary to what we observed in the continuous case, the data does not exhibit a linear trend (on the $\log -\log$ plot). This is due to the fact that in this comparison we are changing $\alpha_{c o n v}$ in each measurement. For a fixed beam current, large pulse widths correspond to a higher number of electrons per pulse. So, while we expect decreasing value of $g^{(2)}(0)$ with electrons per pulse, the factor $\alpha_{\text {conv }}$ also becomes larger, thus, effectively increasing $g^{(2)}(0)$. The model, which accounts for this effect, shows a good agreement with the data. We can therefore extract an excitation efficiency of $\gamma$ $=0.05$. The fact that a lower $\gamma$ is found here compared to the continuous case is fully consistent with the fact that the pulsed experiments were performed with an electron energy of $8 \mathrm{keV}$ instead of $10 \mathrm{keV}$. This choice of lower electron energy allows us to achieve relatively high $g^{(2)}(0)$ amplitudes despite having a high current on the sample ( $214 \mathrm{pA})$ due to the blanking conditions. At this lower electron energy, most bulk plasmons are generated in the top $\mathrm{GaN}$ layer, resulting in fewer excitations close to the quantum wells. The spectrum of the QWs and the Monte Carlo simulations of the electron beam trajectory at $8 \mathrm{keV}$ is provided in the Supporting Information (section S7). Additionally, we can derive the excitation efficiency using eq 10 by simply dividing the area of the bunching peak by the area of any other peak from which we obtain $\gamma=0.06$, which is in good agreement with the value found using the fitting procedure.

\section{ULTRASHORT PULSES}

An extreme case of the model for pulsed $g^{(2)}(\tau)$ measurements is when we have ultrashort electron pulses, that is, in the picosecond regime. In this case, the electron pulse width is very small compared to the emitter lifetime, and thus, the factor accounting for the convolution of both becomes $\alpha_{\text {conv }} \simeq \alpha_{\mathrm{b}}$. Then, eq 9 can be further simplified to

$$
g_{\text {ultrashort }}^{(2)}(0)=1+\frac{b+1}{n_{e} b}=\frac{A_{0}^{p}}{A_{\text {uncorr }, i}^{p}}
$$

where the only remaining parameters are the number of interacting bulk plasmons per electron, which can be also described in terms of excitation efficiency $(\gamma)$, and the average number of electrons per pulse $n_{e}$. In this case, the shape of the bunching peak and, thus, the emitter lifetime, do not contribute to the amplitude of $g^{(2)}(0)$. Moreover, $g^{(2)}(0)$ now can be directly obtained from the ratio between the areas of the different peaks, similar to eq 10 . Hence, when analyzing an experiment, we can simply sum all the counts from each of these two peaks (at $\tau=0$ and $\tau_{i}=i / F, i= \pm 1, \pm 2, \ldots$ ) and divide them to directly obtain the value of $g^{(2)}(0)$. In this way, the analysis to retrieve the excitation efficiency from $g^{(2)}(\tau)$ measurements becomes even simpler. We should also notice that ultrashort pulses are typically achieved by changing the emission statistics of the electron. For example, in the case of photoemission of electron pulses, as in the experiments shown below, the emission of pulses is determined by laser excitation 
of the electron cathode, instead of conventional thermionic or Schottky emission. Our derivation of $g^{2}(0)$ is general and does not assume any particular emission statistics for the electron beam. In the Supporting Information (section S3e) we show a complementary derivation for electron pulses obtained with photoemission.

Figure 4a shows a selection of $g^{(2)}(\tau)$ measurements performed using ultrashort pulses $(\sim 1 \mathrm{ps})$, obtained by

a)
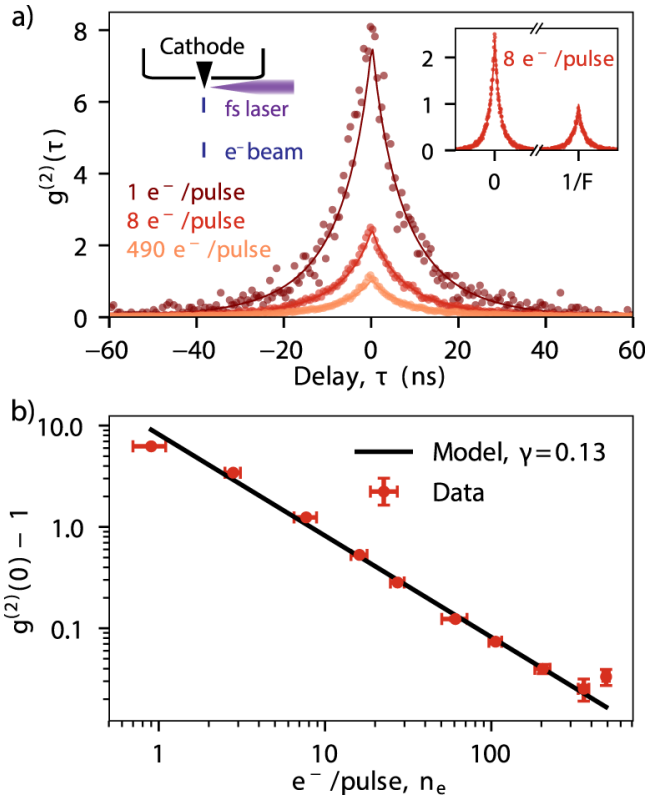

Figure 4. $g^{(2)}(\tau)$ measurements with ultrashort (ps) electron pulses. (a) $g^{(2)}(\tau)$ experiments obtained by changing number of electrons per pulse, with electron energy of $10 \mathrm{keV}$. The experimental data is represented by points, while the solid lines are the fits obtained by solving eq 7 (with $y_{\text {emitter }}(\tau)$ being a stretched exponential). Insets: (left) schematic of the photoemission setup, (right) zoom-out of a $g^{(2)}(\tau)$ measurement, showing that the shape of the uncorrelated peaks (in this case, $\tau=1 / F=200 \mathrm{~ns}$ ) is now determined only by the emitter decay. (b) $g^{(2)}(0)-1$ vs number of electrons per pulse, obtained by dividing the area of the bunching peak by the area of any other peak, as discussed in eq 12. The black solid line corresponds to the best fit obtained using eq 12, which yields an excitation efficiency of $\gamma=0.13$.

focusing a fs laser into the electron cathode at a repetition rate of $5.04 \mathrm{MHz} .{ }^{8,20}$ We chose the conditions for which a larger number of electrons per pulse can be achieved (up to 490 in this case) at the expense of spatial resolution. ${ }^{19}$ This regime allows us to reach the highest possible electron cascade density, as will be discussed below. The experiments were performed with an electron energy of $10 \mathrm{keV}$. The figure shows the 0-delay peak for a changing number of electrons per pulse. We observe that with an average of 1 electron per pulse we obtain $g^{(2)}(0)=7.25$. The right inset in Figure 4a shows a measurement including also the first uncorrelated peak, centered at $\tau=198$ ns. We observe that now both the peaks at $\tau=0$ and at $\tau=1 / F$ have the same shape, determined by the emitter decay. The solid lines again represent the curves obtained by fitting with eq 7 , given that the emitter decay follows a stretched exponential. The best fit is obtained for $\tau_{\text {emitter }}=5.84 \pm 0.07 \mathrm{~ns}$ and $\beta_{\text {emitter }}=0.751 \pm 0.004$. Figure $4 \mathrm{~b}$ shows the value of $g^{(2)}(0)-1$ as a function of the number of electrons per pulse, together with the fit using eq 12 . Here, the data points have been obtained by simply dividing the areas of the bunching peak by the height of peaks at $\tau_{i}=i / F$. The horizontal error bars represent the uncertainty in current measurement in pulsed, partially due to instability in the power of the laser that excites the tip. The vertical error bars are obtained from the analysis of areas below the peaks. We also correct for the fact that the number of events decreases at long delays due to an experimental artifact (see S5). We observe that the data follows the trend predicted by eq 12, yielding the best fit for the model for $\gamma=0.130 \pm 0.001$, which is in agreement with the excitation efficiency found in the experiments in continuous mode, in which the same electron energy was used. This confirms the feasibility of using the $g^{(2)}(\tau)$ analysis with ultrashort electron pulses to obtain the excitation efficiency, thus enabling many applications of $g^{(2)}(\tau)$ spectroscopy in ultrafast electron microscopy.

Even though the data show a linear trend as in the continuous case, we should note that the electron excitation is very different between the continuous and pulsed cases. In ultrafast pulsed mode, we are exciting the sample with a large number of electrons within a very short time (ps), while in the continuous or beam-blanked cases the average time between two consecutive electrons was never smaller than hundreds of ps $(600$ ps at $260 \mathrm{pA})$. We expect that bulk plasmons decay within the first tens of fs after electron excitation, initially creating hot carriers. The thermalization of these carriers typically occurs within tens of ps. ${ }^{5}$ Therefore, in the ps-pulsed $g^{(2)}(\tau)$ experiment up to 490 electrons in each pulse excite the sample within the carrier thermalization time, and in a relatively small area. This raises the question whether we are inducing any nonlinear interaction between carriers due, such as Auger recombination, to high carrier concentrations.

Previous work on InGaN/GaN quantum wells under optical excitation showed that a high excitation fluence leads to a decrease in efficiency, typically referred to as "efficiency droop" ${ }^{33}$ Even though the origin of this effect is still under discussion, some works attributed this efficiency droop to Auger recombination due to locally induced high carrier densities. ${ }^{33}$ Nevertheless, the trend of $g^{(2)}(0)$ with the number of electrons per pulse exhibits a clear power law, as expected from the $g^{(2)}(\tau)$ model, which neglects nonlinear effects. These results suggest that nonlinear interactions between carriers do not play a significant role in this case, even at the highest number of electrons per pulse. This further suggests that the induced carrier densities are lower than the threshold for Auger recombination to occur.

To further elucidate this absence of nonlinear effects, Figure 5a shows CL spectra of the QWs obtained at different number of electrons per pulse. The integrated area below the curve (in the $410-490 \mathrm{~nm}$ spectral range) as a function of the number of electrons per pulse is plotted in the inset of Figure 5a. We observe a clear linear trend with increasing number of electrons per pulse. Figure $5 b$ shows the calculated excess carrier density for a $10 \mathrm{keV}$ electron beam containing 490 electrons. Here we have assumed a radius of the electron beam of $200 \mathrm{~nm}$, corresponding to the expected spatial resolution obtained under our pulsed conditions, calculated using the Fourier transform method explained in ref 19 . We use the Casino software ${ }^{21}$ to estimate the number of inelastic collisions of the electron with the sample. We assume that each collision corresponds to the generation of a bulk plasmon and generates three electron/hole pairs. ${ }^{34,35}$ We observe that the highest carrier density is in the order of $6 \times 10^{17} \mathrm{~cm}^{-3}$. Previous works 


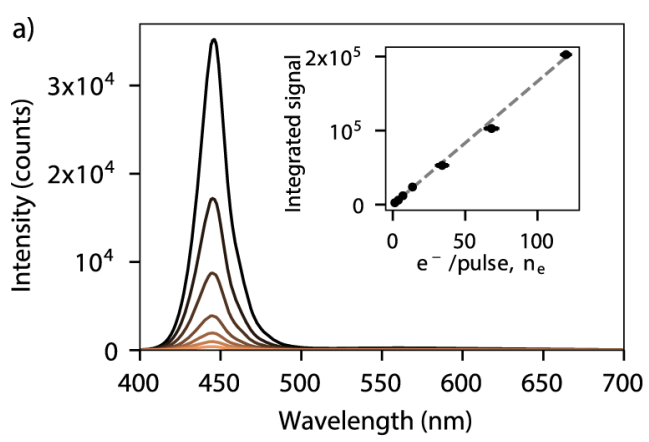

b)

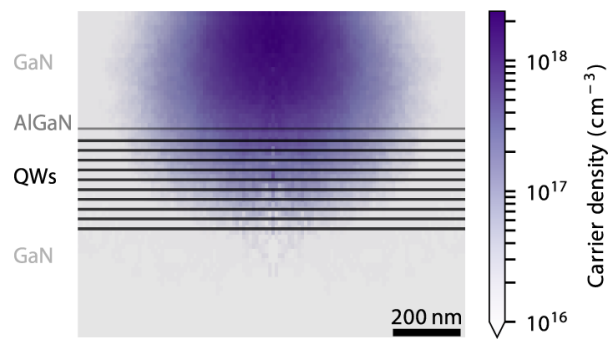

Figure 5. (a) Spectra of the QWs obtained when exciting with ultrashort electron pulses, containing from 1.4 (orange) up to 120 (black) electrons per pulse on average. Inset: integrated signal of the $\mathrm{QW}$ emission as a function of the number of electrons per pulse. The dashed line corresponds to a linear fit. (b) 2D map of the estimated excess carrier density in the sample obtained after excitation of 490 electrons with an energy of $10 \mathrm{keV}$.

based on optical excitation of InGaN show that Auger processes only become dominant for carrier densities larger than $10^{18} \mathrm{~cm}^{-3}$. ${ }^{36,37}$ Therefore, the electron-induced carrier density is below that which would create nonlinear effects. Moreover, we expect the initial spatial distribution of carriers to be relatively localized in space after electron excitation, implying that diffusion of carriers plays a larger role than in optical experiments, in which the spot size is typically larger, as it is limited by diffraction. We note that this is the largest number of electrons per pulse that we can obtain in our system at $10 \mathrm{keV}$. Other pulsed conditions lead to better spatial resolution and, hence, more confined electron cascades, but at a much lower number of electrons per pulse (less than tens of $\mathrm{e}^{-}$per pulse). ${ }^{19}$ Other works have shown a spatial resolution in the nm range but in the regime of a few electrons per pulse and, thus, small electron density. ${ }^{38,39}$

\section{CONCLUSIONS}

In conclusion, we have presented a full description of $g^{(2)}(\tau)$ autocorrelation measurements in incoherent cathodoluminescence spectroscopy for different electron beam configurations. We have developed a fully analytical model to explain the amplitude of bunching $\left(g^{(2)}(0)\right)$ as a function of electron beam current (or number of electrons per pulse), electron excitation efficiency, emitter lifetime, and pulse duration, in the case of pulsed electron beams. The model highlights the inverse dependence of the bunching contribution to $g^{(2)}(\tau)$ as a function of electron beam current or number of electrons per pulse. Moreover, by acquiring a $g^{(2)}(\tau)$ curve at a known electron beam current, we can directly extract the electron excitation efficiency by using a simple equation, and the curve can be fitted to obtain the emitter lifetime. This is a major step forward compared to the previous method in which Monte
Carlo simulations were needed, given the simplicity of the analysis using our model.

In particular, we show that for a pulsed electron beam, the excitation efficiency can be obtained by simply dividing the areas of the peak at 0 delay by that of any other peak, without the need of fitting the data. The model is generic and independent of the sample under study and prior knowledge of the sample geometry. In order to test the model with experiments, we have studied InGaN/GaN quantum well samples, in which we find an excitation efficiency of 0.13 for 10 $\mathrm{keV}$ electrons and 0.05 in the case of $8 \mathrm{keV}$ electrons. Furthermore, we have presented $g^{(2)}(\tau)$ CL measurement using ultrashort (ps) electron pulses, with the average number of electrons ranging from less than 1 to $\sim 500$. The measurements of $g^{(2)}(0)$ as a function of the number of electrons per pulse exhibit the same trend, as predicted by the analytical model, suggesting that nonlinear carrier interactions do not play a role, even at a high number of electrons per pulse. We model the induced carrier density in the QW sample and show that it remains lower than typical values for which nonlinear effects in optical excitation are observed. We foresee that the analytical model will make $g^{(2)}(\tau)$ measurements and analysis more accessible, thus allowing to get deeper insights into the fundamentals of electron-matter interaction. Moreover, the $g^{(2)}(\tau)$ experiments with ultrashort pulses pave the way to study photon statistics with dense electron cascades in a wide range of materials.

\section{ASSOCIATED CONTENT}

SI Supporting Information

The Supporting Information is available free of charge at https://pubs.acs.org/doi/10.1021/acsphotonics.0c01939.

Additional information regarding the analytical model, CL experiments, and data analysis (PDF)

\section{AUTHOR INFORMATION}

\section{Corresponding Author}

Magdalena Solà-Garcia - Center for Nanophotonics, AMOLF, 1098 XG Amsterdam, The Netherlands; ○ orcid.org/0000-0002-2614-1050; Email: m.sola@ amolf.nl

\section{Authors}

Kelly W. Mauser - Center for Nanophotonics, AMOLF, 1098 XG Amsterdam, The Netherlands

Matthias Liebtrau - Center for Nanophotonics, AMOLF, 1098 XG Amsterdam, The Netherlands; 으이.org/00000002-2374-696X

Toon Coenen - Center for Nanophotonics, AMOLF, 1098 XG Amsterdam, The Netherlands; Delmic BV, 2628 EB Delft, The Netherlands; ( ) orcid.org/0000-0002-8043-9798

Silke Christiansen - Fraunhofer Institute for Ceramic Technologies and Systems IKTS, 91301 Forchheim, Germany; () orcid.org/0000-0002-4908-4087

Sophie Meuret - CEMES-CNRS, 31055 Toulouse, France; (1) orcid.org/0000-0001-8511-9972

Albert Polman - Center for Nanophotonics, AMOLF, 1098 XG Amsterdam, The Netherlands; (1) orcid.org/0000-00020685-3886

Complete contact information is available at: https://pubs.acs.org/10.1021/acsphotonics.0c01939 


\section{Notes}

The authors declare the following competing financial interest(s): T.C. is an employee and A.P. is the co-founder and co-owner of Delmic BV, a company that produces the cathodoluminescence system that was used in this work.

\section{ACKNOWLEDGMENTS}

This work is part of the research program of AMOLF which is partly financed by the Dutch Research Council (NWO). This project has received funding from the European Research Council (ERC) under the European Union's Horizon 2020 research and innovation program (Grant Agreement No. 695343) and the FET-Proactive program (grant agreement No. 101017720, EBEAM). S.M. acknowledges support from the French ANR funding agency through the ANR-19-CE300008-ECHOMELO Grant.

\section{REFERENCES}

(1) Meuret, S.; Tizei, L. H. G.; Cazimajou, T.; Bourrellier, R.; Chang, H. C.; Treussart, F.; Kociak, M. Photon Bunching in Cathodoluminescence. Phys. Rev. Lett. 2015, 114 (19), 1-5.

(2) Feldman, M. A.; Dumitrescu, E. F.; Bridges, D.; Chisholm, M. F.; Davidson, R. B.; Evans, P. G.; Hachtel, J. A.; Hu, A.; Pooser, R. C.; Haglund, R. F.; Lawrie, B. J. Colossal Photon Bunching in Quasiparticle-Mediated Nanodiamond Cathodoluminescence. Phys. Rev. B 2018, 97 (8), 1-5.

(3) Lourenço-Martins, H.; Kociak, M.; Meuret, S.; Treussart, F.; Lee, Y. H.; Ling, X. Y.; Chang, H.-C.; Galvão Tizei, L. H. Probing Plasmon-NV0 Coupling at the Nanometer Scale with Photons and Fast Electrons. ACS Photonics 2018, 5, 324-328.

(4) Fox, M. Quantum Optics: An Introduction (Oxford Master Series in Physics); OUP: Oxford, 2006.

(5) Kociak, M.; Zagonel, L. F. Publisher's Note. Ultramicroscopy 2017, 174 (November 2016), 50.

(6) Meuret, S.; Coenen, T.; Zeijlemaker, H.; Latzel, M.; Christiansen, S.; Conesa-Boj, S.; Polman, A. Photon Bunching Reveals Single-Electron Cathodoluminescence Excitation Efficiency in InGaN Quantum Wells. Phys. Rev. B 2017, 96 (3), 1-8.

(7) Meuret, S.; Coenen, T.; Woo, S. Y.; Ra, Y. H.; Mi, Z.; Polman, A. Nanoscale Relative Emission Efficiency Mapping Using Cathodoluminescence g(2) Imaging. Nano Lett. 2018, 18 (4), 2288-2293.

(8) Merano, M.; Sonderegger, S.; Crottini, A.; Collin, S.; Renucci, P.; Pelucchi, E.; Malko, A.; Baier, M. H.; Kapon, E.; Deveaud, B.; Ganière, J.-D. Probing Carrier Dynamics in Nanostructures by Picosecond Cathodoluminescence. Nature 2005, 438 (7067), 479482.

(9) Zewail, A. H. Four-Dimensional Electron Microscopy. Science (Washington, DC, U. S.) 2010, 328 (5975), 187-193.

(10) Polman, A.; Kociak, M.; García de Abajo, F. J. Electron-Beam Spectroscopy for Nanophotonics. Nat. Mater. 2019, 18 (11), 11581171 .

(11) Liao, B.; Najafi, E. Scanning Ultrafast Electron Microscopy: A Novel Technique to Probe Photocarrier Dynamics with High Spatial and Temporal Resolutions. Mater. Today Phys. 2017, 2, 46-53.

(12) Shahmohammadi, M.; Ganière, J. D.; Zhang, H.; Ciechonski, R.; Vescovi, G.; Kryliouk, O.; Tchernycheva, M.; Jacopin, G. Excitonic Diffusion in InGaN/GaN Core-Shell Nanowires. Nano Lett. 2016, 16 (1), 243-249.

(13) Flannigan, D. J.; Barwick, B.; Zewail, A. H. Biological Imaging with 4D Ultrafast Electron Microscopy. Proc. Natl. Acad. Sci. U. S. A. 2010, 107 (22), 9933-9937.

(14) Hanisch-Blicharski, A.; Janzen, A.; Krenzer, B.; Wall, S.; Klasing, F.; Kalus, A.; Frigge, T.; Kammler, M.; Horn-von Hoegen, M. Ultra-Fast Electron Diffraction at Surfaces: From Nanoscale Heat Transport to Driven Phase Transitions. Ultramicroscopy 2013, 127, $2-8$.
(15) Barwick, B.; Flannigan, D. J.; Zewail, A. H. Photon-Induced near-Field Electron Microscopy. Nature 2009, 462 (7275), 902-906.

(16) Feist, A.; Echternkamp, K. E.; Schauss, J.; Yalunin, S. V.; Schäfer, S.; Ropers, C. Quantum Coherent Optical Phase Modulation in an Ultrafast Transmission Electron Microscope. Nature 2015, 521 (7551), 200-203.

(17) Di Giulio, V.; Kociak, M.; de Abajo, F. J. G. Probing Quantum Optical Excitations with Fast Electrons. Optica 2019, 6 (12), 1524.

(18) Brown, R. H.; Twiss, R. Q. Correlation between Photons in Two Coherent Beams of Light. Nature 1956, 177 (4497), 27-29.

(19) Meuret, S.; Solà Garcia, M.; Coenen, T.; Kieft, E.; Zeijlemaker, H.; Lätzel, M.; Christiansen, S.; Woo, S. Y.; Ra, Y. H.; Mi, Z.; Polman, A. Complementary Cathodoluminescence Lifetime Imaging Configurations in a Scanning Electron Microscope. Ultramicroscopy 2019, $197,28-38$.

(20) Solà-Garcia, M.; Meuret, S.; Coenen, T.; Polman, A. ElectronInduced State Conversion in Diamond NV Centers Measured with Pump-Probe Cathodoluminescence Spectroscopy. ACS Photonics 2020, 7 (1), 232-240.

(21) Drouin, D.; Couture, A. R.; Joly, D.; Tastet, X.; Aimez, V.; Gauvin, R. CASINO V2.42 - A Fast and Easy-to-Use Modeling Tool for Scanning Electron Microscopy and Microanalysis Users. Scanning 2007, 29 (3), 92-101.

(22) Ji, Y.; Zhang, Z. H.; Kyaw, Z.; Tiam Tan, S.; Gang Ju, Z.; Liang Zhang, X.; Liu, W.; Wei Sun, X.; Volkan Demir, H. Influence of NType versus p-Type AlGaN Electron-Blocking Layer on InGaN/GaN Multiple Quantum Wells Light-Emitting Diodes. Appl. Phys. Lett. 2013, 103 (5), 1-6.

(23) Suski, T.; Perlin, P.; Teisseyre, H.; Leszczyński, M.; Grzegory, I.; Jun, J.; Boćkowski, M.; Porowski, S.; Moustakas, T. D. Mechanism of Yellow Luminescence in GaN. Appl. Phys. Lett. 1995, 67 (15), 2188-2190.

(24) Julkarnain, M.; Kamata, N.; Fukuda, T.; Arakawa, Y. Yellow Luminescence Band in Undoped GaN Revealed by Two-Wavelength Excited Photoluminescence. Opt. Mater. (Amsterdam, Neth.) 2016, 60, 481-486.

(25) Kimble, H. J.; Dagenais, M.; Mandel, L. Photon Antibunching in Resonance Fluorescence. Phys. Rev. Lett. 1977, 39 (11), 691-695.

(26) Lounis, B.; Bechtel, H. A.; Gerion, D.; Alivisatos, P.; Moerner, W. E. Photon Antibunching in Single CdSe/ZnS Quantum Dot Fluorescence. Chem. Phys. Lett. 2000, 329 (5-6), 399-404.

(27) Morgan, B. L.; Mandel, L. Measurement of Photon Bunching in a Thermal Light Beam. Phys. Rev. Lett. 1966, 16, 1012-1015.

(28) Tan, P. K.; Yeo, G. H.; Poh, H. S.; Chan, A. H.; Kurtsiefer, C. Measuring Temporal Photon Bunching in Blackbody Radiation. Astrophys. J., Lett. 2014, 789 (1), L10.

(29) Bhatti, D.; Von Zanthier, J.; Agarwal, G. S. Superbunching and Nonclassicality as New Hallmarks of Superradiance. Sci. Rep. 2015, 5, $1-8$

(30) Bradac, C.; Johnsson, M. T.; Van Breugel, M.; Baragiola, B. Q.; Martin, R.; Juan, M. L.; Brennen, G. K.; Volz, T. Room-Temperature Spontaneous Superradiance from Single Diamond Nanocrystals. Nat. Commun. 2017, 8 (1), 1205.

(31) Egerton, R. F. Electron Energy-Loss Spectroscopy in the TEM. Rep. Prog. Phys. 2009, 72 (1), 016502.

(32) Lee, K.C. B.; Siegel, J.; Webb, S.E.D.; Leveque-Fort, S.; Cole, M.J.; Jones, R.; Dowling, K.; Lever, M.J.; French, P.M.W. Application of the Stretched Exponential Function to Fluorescence Lifetime Imaging. Biophys. J. 2001, 81 (3), 1265-1274.

(33) David, A.; Young, N. G.; Lund, C.; Craven, M. D. ReviewThe Physics of Recombinations in III-Nitride Emitters. ECS J. Solid State Sci. Technol. 2020, 9 (1), 016021.

(34) Rothwarf, A. Plasmon theory of electron-hole pair production: efficiency of cathode ray phosphors. J. Appl. Phys. 1973, 44, 752.

(35) Keast, V. J.; Scott, A. J.; Kappers, M. J.; Foxon, C. T.; Humphreys, C. J. Electronic Structure of GaN and InxGa1-XN Measured with Electron Energy-Loss Spectroscopy. Phys. Rev. B Condens. Matter Mater. Phys. 2002, 66 (12), 1253191-1253197. 
(36) Shen, Y. C.; Mueller, G. O.; Watanabe, S.; Gardner, N. F.; Munkholm, A.; Krames, M. R. Auger Recombination in InGaN Measured by Photoluminescence. Appl. Phys. Lett. 2007, 91 (14), 141101.

(37) Williams, K. W.; Monahan, N. R.; Koleske, D. D.; Crawford, M. H.; Zhu, X. Y. Ultrafast and Band-Selective Auger Recombination in InGaN Quantum Wells. Appl. Phys. Lett. 2016, 108 (14), 141105.

(38) Sun, J.; Adhikari, A.; Shaheen, B. S.; Yang, H.; Mohammed, O. F. Mapping Carrier Dynamics on Material Surfaces in Space and Time Using Scanning Ultrafast Electron Microscopy. J. Phys. Chem. Lett. 2016, 7 (6), 985-994.

(39) Shahmohammadi, M.; Ganière, J.-D.; Zhang, H.; Ciechonski, R.; Vescovi, G.; Kryliouk, O.; Tchernycheva, M.; Jacopin, G. Excitonic Diffusion in InGaN/GaN Core-Shell Nanowires. Nano Lett. 2016, 16 (1), 243-249. 